\title{
Asymmetric Electron Transfer in Reaction Centers of Purple Bacteria Strongly Depends on Different Electron Matrix Elements in the Active and Inactive Branches
}

\author{
Dmitri Kolbasov ${ }^{\dagger}$ and Avigdor Scherz* \\ Department of Plant Sciences, The Weizmann Institute of Science, Rehovot 76100, Israel
}

Received: June 4, 1999; In Final Form: November 11, 1999

\begin{abstract}
We have re-examined the contribution of electronic matrix elements $\left(V_{1}\right)$ between the primary electron donor and the accessory bacteriochlorophylls in the active (A) and inactive (B) branches of bacterial reaction centers (RC) to the unidirectional light-induced electron transfer (ET) (a preliminary report was recently given by Kolbasov and Scherz in Photosynthesis: Mechanisms and Effects; Garab, G., Ed.; Kluwer Academic Publishers: Dordrecht, 1998; Vol. II; pp 719-722). Our calculations showed that $V_{1_{\mathrm{B}}}{ }^{2}$ is probably smaller by 3 orders of magnitude than $V_{1_{\mathrm{A}}}{ }^{2}$ in Rb. sphaeroides and by at least 1 order of magnitude in Rps. viridis. These phenomena reflect the quantum interference and mutual cancellation of the resonance integrals corresponding to different ET pathways between atoms of $\mathrm{P}$ and the accessory bacteriochlorophyll in the inactive branch. The calculated values of $V$ and the corresponding ET rate constants for mutated RC further support this conclusion. Zhang and Friesner (Proc. Natl. Acad. Sci. U.S.A. 1998, 95, 13603-13605), using more elaborate calculations, showed that $V_{\mathrm{A}_{\mathrm{A}}} / V_{\mathrm{l}_{\mathrm{B}}}$ for Rps. viridis can reach a value of 14 for same reason, indicating that differences in the overlap matrix elements are key factors in the unidirectional electron flow in both organisms.
\end{abstract}

\section{Introduction}

In photosynthetic reaction center (RC) light induces ET from a bacteriochlorophyll (BChl) dimer termed the special pair (P), to an initial acceptor, bacteriopheophytin $(\mathrm{H})$, through an intermediate $\mathrm{BChl}$ molecule called the accessory bacteriochlorophyll. The RC contains two accessory BChls $\left(\mathrm{B}_{\mathrm{A}}\right.$ and $\left.\mathrm{B}_{\mathrm{B}}\right)$ and two bacteriopheophytins in approximately 2-fold symmetry. Yet ET always proceeds along one branch (termed the "A" branch). ${ }^{1-3}$

According to the nonadiabatic theory of ET, the rate of the reaction is proportional to the product of two independent terms: the nuclear (or Franck-Condon) factor and the square of the electronic matrix transfer element. ${ }^{4}$ The Franck-Condon factor (FC) is associated with the energetics of the ET reaction and represents the probability of nuclear conformations allowing electronic transitions among molecules with particular redox potentials. The electronic matrix element, $V$, measures the coupling between the electron wave functions of the donor and the acceptor. It determines the probability of an electronic transition occurring at a favorable medium conformation.

Theoretical and experimental studies thus far have concentrated mostly on the FC term, assuming that the unidirectional electron flow relies on differences in the energies of states $\mathrm{P} * \mathrm{BH}, \mathrm{P}^{+} \mathrm{B}^{-} \mathrm{H}$, and $\mathrm{P}^{+} \mathrm{BH}^{-}$in the two branches. ${ }^{5-9}$ Asymmetrical charge distribution between the two halves of $\mathrm{P}^{*}$, resulting from differences in the protein environment, was also proposed to have a role in the unidirectional ET. $6,7,10-12$ However, mutated RC, in which one of the BChls comprising the special pair is replaced by the more oxidative bacteriopheophytin (thus forming the so-called "hetero dimer"), did not show electron transfer in the B branch.

Although the role of $V$ in controlling the ET asymmetry has attracted less attention, several recent studies have pointed out

* Corresponding author. E-mail: bcscherz@weizmann.weizmann.ac.il. Fax: (972)-8934-4181.

† In partial fulfillment of $\mathrm{Ph}$. D. Thesis. differences between the calculated $V_{\mathrm{A}}$ and $V_{\mathrm{B}}$ that could significantly contribute to the observed asymmetry. ${ }^{8,13-15}$ Here we have re-examined $V$ for $\mathrm{P} / \mathrm{B}_{\mathrm{A}}$ and $\mathrm{P} / \mathrm{B}_{\mathrm{B}}$ using the coordinates of $R b$. sphaeroides, at a resolution of $2.2 \AA,{ }^{16}$ and of Rps. viridis at a resolution of $2.3 \AA^{17}$ from the Protein Data Bank. ${ }^{18}$

Electronic couplings (EC) are classified either as throughspace ECs or through-bond ECs. ${ }^{19}$ The through-bond coupling can be calculated by using the standard sets of basis functions of quantum chemistry. These basis functions have been specifically developed for the reliable determination of the electronic properties of chemically bound atoms. Thus, they provide a reasonable representation of the electronic wave functions at distances (from the nuclei) that are smaller or equal to the bond length. However, these standard basis sets are not designed for calculating the coupling through an interval of space that is larger than the bond length. In particular, they fail to represent the tails of the wave functions. Although these tails contribute only slightly to the energies of the electronic states and spectra, they are key factors in determining the through-space electron transfer. If the distance dependence has not been properly considered, the through-space couplings appear to be underestimated, and thus, the role of the through-bond couplings may be overestimated.

The EC for the initial ET in the bacterial RC probably occurs through space, because no protein matter fills the closest pathway between the tetrapyrrole molecules of the RC. Here, the details of the electronic wave function tails are of prime importance. In one approach, $V^{2}(R)$ is deduced from the logarithmic dependence of the electron-transfer rate, $k_{\mathrm{ET}}$ on $R$, where $R$ is the distance between the molecules of the donor and acceptor for many donor/acceptor pairs, ${ }^{20}$

$$
V^{2} \approx \exp (-\gamma R)
$$

where $\gamma$ is the decay parameter. Curry et al. ${ }^{21}$ obtained a value of 1.47 for $\gamma$ based on pathways through bonded atoms. Moser et al. ${ }^{20}$ suggested a value of 1.4 based on experimental ET rates 
in proteins. Expression 1 is probably oversimplified because it does not consider the forms, symmetries, and relative orientations of the interacting orbitals. In through-space coupling, the symmetries of the coupled orbitals are of prime importance. For example, if the wave functions of the coupled molecules consist of alternating positive and negative parts, their products also consist of alternating domains that may cancel each other. The overlap value critically depends on the relative positions of the molecules.

The complicated dependence of the molecular couplings (MC) on the relative positions of the donor and acceptor is difficult to deduce from the empirical rate constants. In fact, some properties of the couplings should be predefined on a theoretical basis. One such approach is to use the LCAO approximation, with $\mathrm{MC}$ being the linear combination of atomic couplings (AC) summed over all pairs of atoms, weighted by the atomic coefficients of the atoms in the donor and acceptor molecular orbitals (MO). This approach accounts for the forms and symmetries of the MOs involved. The angular dependence for ACs may be taken from theory, whereas the distance dependence fits to the experimental data. Warshel and Parson and Alden et al. ${ }^{22,23}$ suggested several semiempirical expressions for ACs that are partially based on empirical data for donors and acceptors at relatively large separations.

Although semiempirical equations for the distance dependence of ACs may be very useful, they cannot be derived in a straightforward way from the experimentally available distance dependence of MCs. Importantly, MCs always decay faster than the ACs from which they are constructed, the same way as the electrostatic interaction between dipoles decreases faster than that of single charges.

The other approach is to calculate the couplings entirely on theoretical grounds. Scherer et al. ${ }^{8}$ used slowly decaying wave functions in combination with INDO/S calculations, but the details of these calculations were not reported. Some calculations that are not based on the LCAO approximation were proposed, ${ }^{24,25}$ but they failed to describe the molecular orbitals to a good precision. In 1986, Kühn suggested a method based on LCAO and a different set of atomic wave functions. ${ }^{26}$ A major advantage of these wave functions was their relatively slow decay. Unfortunately, at the time Kühn performed his original calculation, the atomic structure of $\mathrm{RC}$ had not been determined to a sufficient resolution, and to our knowledge Kühn never applied his method to a better resolved structure. The recent release of new coordinates, combined with the realization of the physical drawbacks in current calculations motivated us to reapply Kühn's type calculations (with a few modifications) to $\mathrm{RC}$ from wild type and mutated Rps. viridis and Rb. sphaeroides. We ignored the contribution of hyperconjugated saturated groups (such as methyls) to $V$, because there is still no agreement whether the hyperconjugated groups play any significant role in the intermolecular couplings in RC. The opinions vary from total neglect of the hyperconjugated group ${ }^{22}$ to assigning to them the main contribution. ${ }^{14}$ Also it is not clear whether hyperconjugated groups facilitate the MCs by extending the MOs ${ }^{14}$ or the "hole transfer" through occupied hyperconjugated groups contributes negative components to the MC. ${ }^{27} \mathrm{We}$ assume that when the through-space atomic couplings are properly considered, the extension of the MOs is not necessary for having a reasonable estimate of the coupling.

\section{Methods}

This section presents the calculation of the matrix elements for ET among P and B for bacterial RCs. The calculation relies on the method suggested by $\mathrm{H}$. Kühn, ${ }^{26}$ with a few modifications. It considers three electronic states in the $\mathrm{RC}:\left|\mathrm{P}^{*} \mathrm{BH}\right\rangle$, $\left|\mathrm{P}^{+} \mathrm{B}^{-} \mathrm{H}\right\rangle$, and $\left|\mathrm{P}^{+} \mathrm{BH}^{-}\right\rangle$. The matrix elements for the first successive step of the initial ET were defined as

$$
V_{1}=\left\langle\mathrm{P}^{+} \mathrm{B}^{-} \mathrm{H}\left|\mathrm{H}_{1}^{\prime}\right| \mathrm{P}^{*} \mathrm{BH}\right\rangle
$$

where $\mathrm{H}^{\prime}$ represents a perturbation that mixes the states. We assumed that in the simplified, one-electron treatment, the wave functions of $\left|\mathrm{P}^{*} \mathrm{BH}\right\rangle,\left|\mathrm{P}^{+} \mathrm{B}^{-} \mathrm{H}\right\rangle$, and $\left|\mathrm{P}^{+} \mathrm{BH}^{-}\right\rangle$can be represented as the LUMOs of $\mathrm{P}, \mathrm{B}$, and $\mathrm{H}$, respectively. The LUMOs were calculated as linear combinations of the atomic $\mathrm{p}$ orbitals. The matrix element is presented by a linear combination of the twoatom resonance integrals $S_{i j}$

$$
V=\sum_{i} \sum_{j} b_{i} b_{j} S_{i j}
$$

where $b_{i}$ and $b_{j}$ are the atomic LCAO coefficients for the LUMO of the donor and acceptor, respectively.

Kühn suggested using linear combination of atomic orbitals centered on the antinodes of the molecular wave function. However, we used a combination of atomic orbitals centered on atoms, since this method has higher flexibility in considering the conjugated branch chains of the BChls. For calculating the AC, we used the simple Hückel set of coefficients, ${ }^{28}$ because they usually yield the same electronic distribution as that obtained using more sophisticated methods, ${ }^{27}$ such as the ab initio calculation. ${ }^{29}$

Calculation of the resonance integrals using Kühn's method is described in the Appendix. The calculated integrals depend on two parameters: the ionization potential of the donor in the medium, $I$, and the fast (optical) dielectric constant, $D_{\mathrm{e}}$. The value of $D_{\mathrm{e}}$ should be corrected by the local-field factor, $f^{2}{ }^{23,30}$ Since $f^{2} / D_{\mathrm{e}}$ is not very different from 1 , we substituted the value of 1 for $D_{\mathrm{e}}$.

The ionization potential, $I$, was deduced from the redox potential measurements of RC. The ground-state redox potential of $\mathrm{P}$ in $\mathrm{RC}$ of $R b$. sphaeroides and Rps. viridis versus the normal hydrogen electrode $(\mathrm{NHE}), E^{\mathrm{NHE}}\left(\mathrm{P} / \mathrm{P}^{+}\right)$, is about $0.50 .{ }^{31-33}$ Hence, the ionization potential of $\mathrm{P}$ in the first excited singlet state is ${ }^{34}$

$$
I=0.5+4.44-h v / e+\Delta \Delta G_{\mathrm{sol}}
$$

where $\Delta \Delta G_{\text {sol }}$ is the difference between the solvation energies of neutral and ionized $\mathrm{P}$ within the protein matrix, $h$ is Planck's constant, $v$ is the ${ }^{1} \mathrm{~S}_{0-0}$ frequency of $\mathrm{P}, 4.44 \mathrm{eV}$ is the NHE ionization potential in a vacuum, ${ }^{35}$ and $e$ is the elementary charge value (positive, in esu units). As already mentioned, the differences in solvation energies of similar cofactors of the A and $\mathrm{B}$ branches were recently considered to be important for the unidirectionality of the ET. ${ }^{7-9}$ However, in calculating the ionization potentials, we can neglect these differences because $\Delta \Delta G_{\text {sol }}$ is proposed to be of the order of $0.1 \mathrm{eV}, 7,36,37$ which is not important for the matrix element calculation. Using eq $4, I$ was assigned a value of $3.5 \mathrm{eV}$.

Substituting the assigned values of $D_{\mathrm{e}}$ and $I$ in eqs A6 and A7 and the values of $a$ and $n$ into eq A9 of the Appendix, we obtain the following formula for the interatomic resonance integral for the first step of ET in bacterial $\mathrm{RC}$, if $\mathrm{P}$ is the donor and $\mathrm{B}$ is the acceptor (in $\mathrm{eV}$ )

$$
\begin{aligned}
& S_{i j}(\mathrm{~PB})=25 \exp \left(-0.96 d_{j i}\right) d_{j i}{ }^{0.97}\left\{-\cos Z_{i} \cos Z_{j}+\right. \\
& \left.1.04 d_{j i}{ }^{-1}\left(\cos X_{i} \cos X_{j}+\cos Y_{i} \cos Y_{j}\right)\right\}
\end{aligned}
$$


where $d_{j i}$ is the interatomic distance $(\AA), \cos X_{i}$ is the $x$-direction cosine of the p-orbital of the $i$ th atom; the $z$ axis points along the line connecting the two atoms. Following this approach, we considered $\mathrm{P}^{*}$ to be a single donor in calculating $V_{1}$. The resonance interaction between its two monomeric halves splits the two pairs of orbitals (LUMOs and HOMOs) into four. ${ }^{38}$ In particular, the LUMO splits into two combinations, $(+)$ and $(-)$, where $\operatorname{LUMO}(+)$, the lower energy one, is given by

$$
\Phi_{\mathrm{P}}=c_{\mathrm{A}} \Phi_{\mathrm{P}_{\mathrm{A}}}+c_{\mathrm{B}} \Phi_{\mathrm{P}_{\mathrm{B}}}
$$

where $\Phi_{\mathrm{P}_{\mathrm{A}}}$ and $\Phi_{\mathrm{P}_{\mathrm{B}}}$ are the monomers' LUMOs. The probability of finding the excited electron within the $i$ th monomer is $c_{i}{ }^{2}$. Thus, the matrix elements for the first ET step in the A and B branches are

$$
\begin{aligned}
& V_{1_{\mathrm{A}}}=c_{\mathrm{A}} V_{\mathrm{P}_{\mathrm{A}} \mathrm{B}_{\mathrm{A}}}+c_{\mathrm{B}} V_{\mathrm{P}_{\mathrm{B}} \mathrm{B}_{\mathrm{A}}} \\
& V_{1_{\mathrm{B}}}=c_{\mathrm{A}} V_{\mathrm{P}_{\mathrm{A}} \mathrm{B}_{\mathrm{B}}}+c_{\mathrm{B}} V_{\mathrm{P}_{\mathrm{B}} \mathrm{B}_{\mathrm{B}}}
\end{aligned}
$$

where $V_{\mathrm{P}_{\mathrm{A}} \mathrm{B}_{\mathrm{A}}}$ is the coupling of the monomer $\mathrm{P}_{\mathrm{A}}$ to $\mathrm{B}$ in the active branch. The resonance interaction between the monomers is as large as $0.15-0.2 \mathrm{eV},{ }^{39,40}$ which is large enough to consider $\mathrm{LUMO}(+)$ as a single coherent state.

The distribution of electron densities among the $\mathrm{A}$ and $\mathrm{B}$ monomers of $\mathrm{P}^{*}$ is controversial. ${ }^{11,22,38,41-43} \mathrm{We}$ assumed that in the wild type RC the excited electron is equally distributed. Interaction with an external electric field makes the electron distribution in $\mathrm{P}^{*}$ nonsymmetrical ${ }^{8}$ because of different potentials created by this field at $\mathrm{P}_{\mathrm{A}}$ and $\mathrm{P}_{\mathrm{B}}$. The potentials in the field of charged or polar residues are calculated by considering atoms as point charges screened by the medium, which is represented by the slow (nuclear) dielectric constant. Alden et al. ${ }^{23}$ proposed that the slow dielectric constant at a distance $r$ from the charge is given by

$$
D(r)=D+(1-D) \exp -\left(r / r_{\mathrm{o}}\right)^{3}
$$

where $r_{\mathrm{o}}$ is equal to $4 \AA$, and $D$ is the macroscopical dielectric constant corresponding to infinite distance. Thus, the effect of the environmental charges $q_{n}$ on the energy of a particular MO orbital is

$$
F=-e \sum_{n} q_{n} \sum_{i} b_{i}\left\{r_{i n} D\left(r_{i n}\right)\right\}^{-1}
$$

where $b_{i}$ represent the orbital coefficients corresponding to the $i$ th atom, $r_{i n}$ is the distance between the $i$ th atom and the $n$th charge, and $e$ is the elementary charge (in esu units, positive).

For deriving the polarizability of the $\operatorname{LUMO}(+)$ of $\mathrm{P}$, we used the simple Hückel approach as described by Artz et al. ${ }^{40}$ The LUMOs of the two monomers were considered to be two one-electron states having nondiagonal coupling matrix elements, $\beta$. The eigenenergies of the monomeric LUMOs are $E_{\mathrm{A}}$ and $E_{\mathrm{B}}$. Artz et al. (1997) suggested that $\beta=170 \mathrm{meV}$ (for the two HOMOs) in $R b$. sphaeroides. Similiar values were suggested by Fischer and Scherer ${ }^{39}$ and Warshel and Parson ${ }^{22}$ for Rps. viridis. Here, we used the value of $170 \mathrm{meV}$ for the LUMOs of both species. The resulting $2 \times 2$ Hamiltonian matrix was diagonalized to obtain the coefficients $c_{\mathrm{A}}$ and $c_{\mathrm{B}}$ of the LUMO(+). We assumed that in the wild type RC $E_{\mathrm{A}}$ and $E_{\mathrm{B}}$ were equal, and in the modified RC one of these energies was changed by $\Delta \alpha<0$. It was also assumed that in heterodimers $\Delta \alpha=-0.2 \mathrm{eV}$, which is the difference between the reduction potentials of BPha and BChla. ${ }^{34}$ In M201 Gly $\rightarrow$ Asp mutant, $\Delta \alpha$ was calculated as the difference of the electrostatic potentials
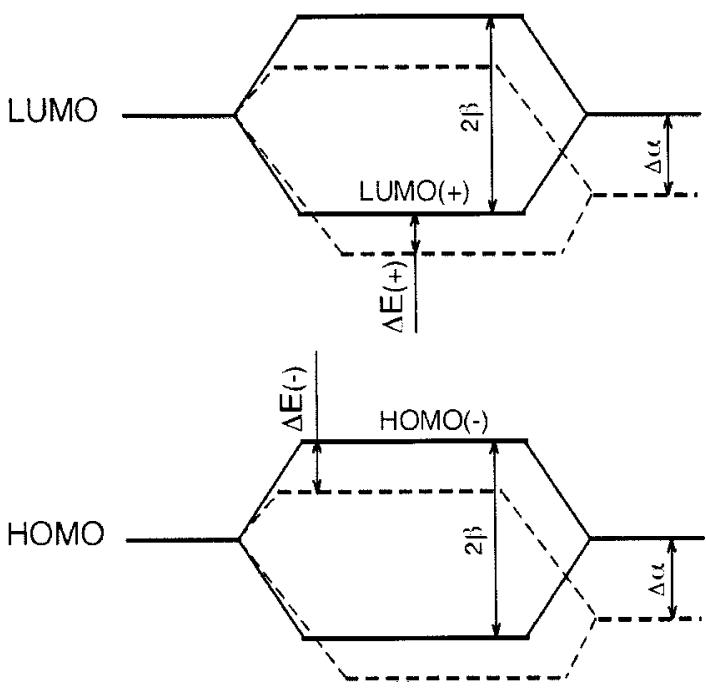

Figure 1. Splitting of the monomers' orbitals of $\mathrm{P}$ in the wild type (solid) and modified (dashed) RC.

created by the charged Asp residue, using eq 9. In M210 Tyr $\rightarrow$ Phe and L168 His $\rightarrow$ Phe, $\Delta \alpha$ was calculated from the change of the oxidation potential caused by the mutation, $\Delta E_{(\mathrm{OX})}$. We assumed that the energies of the HOMO and LUMO of the monomer change by the same value, $\Delta \alpha$, and the couplings between the HOMOs and the LUMOS, $\beta$, are both equal to $170 \mathrm{meV}$. Figure 1 presents the corresponding scheme of the energy levels, where LUMOs and HOMOs are split into two pairs of eigenstates. The eigenenergy, $E_{(+)}$, of the $\operatorname{LUMO}(+)$, corresponds to the reduction potential of $\mathrm{P}$, and the eigenenergy of the $\mathrm{HOMO}(-), E_{(-)}$, corresponds to the oxidation potential: $\Delta E_{(-)}=-e \Delta E_{(\mathrm{OX})}, \Delta E_{(+)}=-e \Delta E_{(\mathrm{RED})}, \Delta E_{(\mathrm{OX})}$, and $\Delta E_{(\mathrm{RED})}$ are the changes of the oxidation and reduction potentials, respectively and $e$ is the elementary charge (in esu units, positive).

$$
\Delta E_{(+)}=\Delta \alpha / 2+\beta-\left(\Delta \alpha^{2} / 4+\beta^{2}\right)^{1 / 2}
$$

The value of $\Delta \alpha$ can be calculated from $\Delta E_{(-)}$by substituting $\Delta \alpha-\Delta E_{(-)}$for $\Delta E_{(+)}$in eq 10

$$
\Delta \alpha=\Delta E_{(-)}\left(\Delta E_{(-)}+2 \beta\right) /\left(\Delta E_{(-)}+\beta\right)
$$

Importantly, at the present level of calculations, the model assumes that $\left|\Delta E_{(-)}\right|<\beta$ and $\mathrm{B}_{\mathrm{A}}$ is not significantly affected. A decrease of $\Delta E_{(+)}$then results in the equal increase of the $\Delta G$ for the ET: $\Delta \Delta G=-\Delta E_{(+)} .=\Delta \alpha-\Delta E_{(-)}$. According to the Marcus equation, ${ }^{44,45}$ the rate constant, $k_{\mathrm{ET}}$, is

$$
k_{\mathrm{ET}}=4 \pi^{2} / h V^{2} \mathrm{FC}
$$

where the Franck-Condon factor, FC, in the high-temperature limit, depends on $\Delta G$ as follows:

$$
\mathrm{FC}=(4 \pi \lambda k T)^{-0.5} \exp \left\{-(\Delta G+\lambda)^{2} /(4 \lambda k T)\right\}
$$

Here, $\lambda$ is the reorganization energy, $k T$ is the Bolzmann factor, and $h$ is Planck's constant. Recent measurements by Volk et al. ${ }^{46}$ and the calculation of Sim and Makri ${ }^{47}$ suggested that $\Delta G_{\mathrm{WT}}$ for the first step of ET in the wild type $R b$. sphaeroides is $-0.05 \mathrm{eV}$. We assumed that the ET in the wild-type RC is activationless; thus, the reorganization energy $\lambda=-\Delta G_{\mathrm{WT}}$. Substituting these values and $\Delta E_{(+)}$(in eV) into (13) and assuming that in the wild-type $\mathrm{RC} E_{\mathrm{A}}$ and $E_{\mathrm{B}}$ are equal, the change of the FC factor because of mutation is given by (for 
TABLE 1: Calculated Matrix Elements, $V_{1}(\mathrm{meV})$ for Bacterial Reaction Center Coordinates

\begin{tabular}{ccc}
\hline coupled molecules & Rps. viridis & Rb. sphaeroides \\
\hline $\mathrm{P}_{\mathrm{A}} \rightarrow \mathrm{B}_{\mathrm{A}}$ & 2.19 & -3.82 \\
$\mathrm{P}_{\mathrm{B}} \rightarrow \mathrm{B}_{\mathrm{A}}$ & 8.51 & 10.42 \\
$\mathrm{P} \rightarrow \mathrm{B}_{\mathrm{A}}{ }^{a}$ & 7.57 & 4.67 \\
$\mathrm{P}_{\mathrm{A}} \rightarrow \mathrm{B}_{\mathrm{B}}$ & 4.13 & -4.47 \\
$\mathrm{P}_{\mathrm{B}} \rightarrow \mathrm{B}_{\mathrm{B}}$ & -0.05 & 4.68 \\
$\mathrm{P} \rightarrow \mathrm{B}_{\mathrm{B}}{ }^{a}$ & 2.89 & 0.15
\end{tabular}

${ }^{a}$ The calculations assume equal contributions of the two halves of $\mathrm{P}, c_{\mathrm{A}}=c_{\mathrm{B}}=1 / \sqrt{ } 2$.

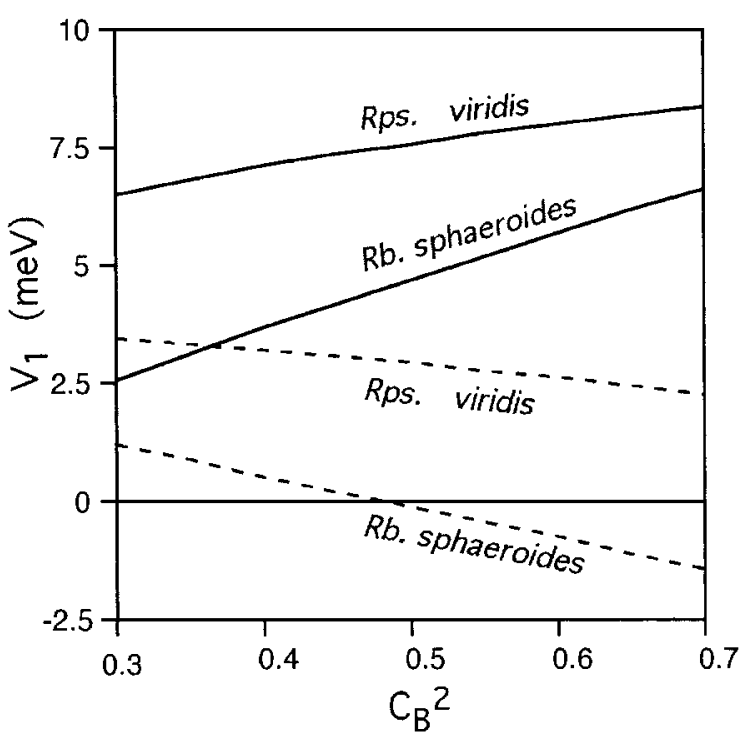

Figure 2. The electronic matrix elements, $V_{1}$, in the active (solid) and inactive (dashed) branches of $\mathrm{RC}$, as a function of the electron density distribution, $C_{\mathrm{B}}{ }^{2}$, in the LUMO of $\mathrm{P}$.

room temperature)

$$
\mathrm{FC} / \mathrm{FC}_{\mathrm{WT}}=\exp \left\{-\left[\Delta \alpha-\Delta E_{(-)}\right]^{2}\right\} / 0.005
$$

If $E_{\mathrm{A}}$ or $E_{\mathrm{B}}$ increases, the ET process is in the inverted Marcus region. ${ }^{44,45}$ No further calculations were carried out for FC in this region.

\section{Results}

Table 1 presents the calculated matrix elements $V_{1}$ for lightinduced electron transfer between $\mathrm{P}^{*}, \mathrm{~B}_{\mathrm{A}}$, and $\mathrm{B}_{\mathrm{B}}$, in Rps. viridis and $R b$. sphaeroides for symmetrical electron distribution in the two monomeric halves. Figure 2 illustrates the dependence of the calculated matrix elements on the distribution of electron density in the $\operatorname{LUMO}(+)$. Figure 3 presents the dependence of $V_{1_{\mathrm{A}}}$ on the distance between $\mathrm{P}$ and $\mathrm{B}_{\mathrm{A}}$. Here, $\mathrm{B}_{\mathrm{A}}$ has been translated along the axis connecting the geometrical centers of $\mathrm{P}$ and $\mathrm{B}_{\mathrm{A}}$. Interestingly, for this particular coordinate $V_{1_{\mathrm{A}}}$ appears to have a shallow maximum around the native configuration of pigments.

For experimental proof of our calculations, we were looking for mutations that can change the electron density distribution between the two halves of $\mathrm{P}^{*}$. These include formation or breakage of a hydrogen bond to $\mathrm{P}$, formation of a heterodimer by substitution of $\mathrm{BPh}$ for one of the monomers of $\mathrm{P}$, or polarization of $\mathrm{P}$ by charged or polar groups.

Table 2 presents empirical and predicted ratios between the rate constants for mutated and wild-type RCs, considering changes in $V_{1}$. Here $c_{\mathrm{B}}{ }^{2}$ is the squared coefficient of $P_{\mathrm{B}}$ in the $\mathrm{LUMO}(+), c_{\mathrm{A}}^{2}+c_{\mathrm{B}}{ }^{2}=1$. In calculating the ratio between the rate constants of electron transfer in the wild type, heterodimer containing mutants, and RC mutated at the M210 position, we estimated the changes in the FC using eq 14. In calculating FC, we ignored the fact that here $V$ is not independent of nuclear motions. Two mutations were reported to promote ET in the B branch (to a maximum value of about $10-15 \%$ of the active branch of the mutant). Table 3 shows the reported results and our predicted rate constants for these mutants.

The values in Tables 2 and 3 were calculated as follows. The mutants under discussion may be divided into two groups. The first group consists of mutations of M210 and M201 (M203 in $R b$. sphaeroides). These mutations modify the electric field around $\mathrm{P}$ and thereby strongly affect the polarization of $\mathrm{P}^{*}$ without changing its coordination or hydrogen bonding to the protein matrix. In Phe-M210, the $\mathrm{OH}$ dipole of Tyr is missing, consequently decreasing the energy of $\operatorname{LUMO}(+)$. Since this dipole is positioned close to $\mathrm{P}_{\mathrm{A}}{ }^{*}$, we think that this mutation reduces the energy of $\mathrm{P}_{\mathrm{A}}$ *. The Asp-M203 probably introduces a negatively charged residue closer to $\mathrm{P}_{\mathrm{B}} *$ than to $\mathrm{P}_{\mathrm{A}}$ *, shifting the electron density to $\mathrm{P}_{\mathrm{A}}$. Czarnecki et al. ${ }^{48}$ have provided some spectroscopic evidence that the Asp is ionized. Although the negative Asp should destabilize $\mathrm{P}$, the observed oxidation potential in this mutant is the same as in the wild type, ${ }^{49,50}$ indicating an additional stabilization that compensates for the electrostatic repulsion. The energy difference between the two halves of $\mathrm{P}^{*}$ in this mutant was calculated using eqs 8 and 9 , and a value of 5 for $D .{ }^{51}$ In calculating the electrostatic effect of the mutation, we positioned the Asp within the protein matrix of $R b$. sphaeroides so that sterical hindrance with nearby residues was minimal. The negative charge was equally distributed between the carboxylic oxygen atoms.

The second group consists of mutants in which well-defined chemical modification was the reason for the energy difference between the monomeric LUMOs. In L173 His $\rightarrow$ Leu and M200/202 His $\rightarrow$ Leu, ${ }^{52,53}$ the mutation resulted in substituting $\mathrm{BPh}$ for BChl. The other mutant of this group is L168 His $\rightarrow$ Phe. ${ }^{54}$ Here, the oxidation potential is known. After estimating $c_{\mathrm{B}}{ }^{2}$, we derived the corresponding matrix elements from Figure 2 , using in the case of $R b$. capsulatus the values calculated for Rb. sphaeroides.

\section{Discussion}

Most current studies suggest that differences in the redox energies of the electron carriers in the two branches of RC account for the unidirectional electron flow. Here, we reexamined the theoretical contribution of differences in the overlap matrix elements. Our calculations show that $V_{1_{\mathrm{B}}}{ }^{2}$ is probably smaller by 3 orders of magnitude than $V_{1_{\mathrm{A}}}^{2}$ in $R b$. sphaeroides and by at least 1 order of magnitude in Rps. viridis. The most interesting result of these calculations is the cancellation of $V_{1_{\mathrm{B}}}$ in $R b$. sphaeroides because of the quantum interference and mutual cancellation of the resonance integrals corresponding to different ET pathways between atoms from $\mathrm{P}$ and $\mathrm{B}$ (Table 1). Zhang and Friesner ${ }^{15}$ showed by more elaborate calculations that $V_{1_{\mathrm{A}}} / V_{1_{\mathrm{B}}}$ for Rps. viridis can reach a value of 14 for the same reason, indicating that the differences in the overlap matrix elements are key factors regarding the unidirectional electron flow in both organisms.

The difference of the matrix elements in the $\mathrm{A}$ and $\mathrm{B}$ branches reflects two main features of the bacterial reaction centers. (1) The difference in the cofactors' positions, which do not fully match in the two species. In Rps. viridis, the asymmetry may be described as a $5^{\circ}$ rotation of $\mathrm{P}$ around an axis through the centers of $\mathrm{B}_{\mathrm{A}}$ and $\mathrm{B}_{\mathrm{B}}$, relative to the symmetry of the $\mathrm{P}, \mathrm{B}_{\mathrm{A}}$, 


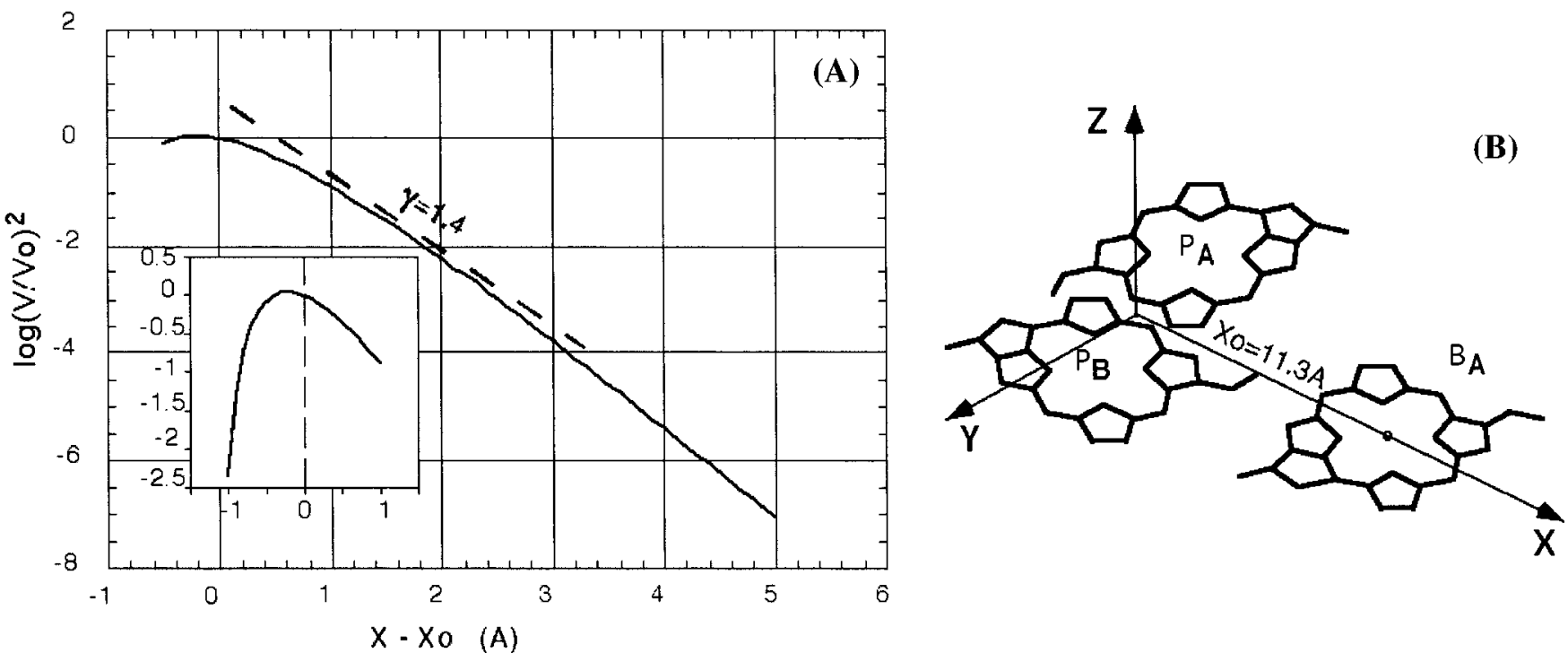

Figure 3. (A) Distance dependence of $V_{1_{\mathrm{A}}}$ calculated for $R b$. sphaeroides using eqs 3 (solid line) and 1 (dashed line). (B) Translation direction (axis $X$ ) of the accessory $\mathrm{BChl}, \mathrm{B}_{\mathrm{A}}$. The origin of the coordinate system is placed at the midpoint of the line connecting the two $\mathrm{Mg}$ atoms of $\mathrm{P}_{\mathrm{A}}$ and $\mathrm{P}_{\mathrm{B}}$, and the $X$ axis is on the line connecting $X_{0}$ and the $\mathrm{Mg}$ of $\mathrm{B}_{\mathrm{A}}$.

TABLE 2: Experimental and Calculated Parameters for ET in the Active Branch

\begin{tabular}{|c|c|c|c|c|c|c|c|c|c|}
\hline mutation & species & $\begin{array}{c}\Delta \alpha \\
(\mathrm{meV})\end{array}$ & $\begin{array}{l}\Delta E_{(-)} \\
(\mathrm{meV})\end{array}$ & $\begin{array}{l}\Delta E_{(+)} \\
(\mathrm{meV})\end{array}$ & $C_{\mathrm{B}}^{2}$ & $\left(V_{\mathrm{mut}}\right)^{2} /\left(V_{\mathrm{WT}}\right)^{2}$ & $\begin{array}{c}k_{\mathrm{mut}} / k_{\mathrm{WT}} \\
\text { calcd }^{b}\end{array}$ & $\begin{array}{c}k_{\mathrm{mut}} / k_{\mathrm{WT}} \\
\text { exptl }\end{array}$ & $\operatorname{ref}^{c}$ \\
\hline M210 Tyr $\rightarrow$ Phe & $\operatorname{spha}^{e}$ & -65 & $-30^{\mathrm{a}}$ & -35 & 0.4 & 0.56 & $0.44^{d}$ & $0.3-0.4$ & $59-61$ \\
\hline \multirow[t]{2}{*}{ M203/201 Gly $\rightarrow$ Asp } & spha $^{e}$ & $+100^{a}$ & 0 & & 0.36 & 0.42 & & 0.36 & 49,50 \\
\hline & caps $^{f}$ & $+100^{a}$ & 0 & & 0.36 & 0.42 & & & 0.2 \\
\hline L168 His $\rightarrow$ Phe & $\operatorname{vir}^{g}$ & +135 & $+80^{a}$ & & 0.69 & 1.5 & & 3.2 & 54 \\
\hline \multirow[t]{2}{*}{ M200/202 His $\rightarrow$ Leu } & caps $^{f}$ & $-200^{a}$ & & -125 & 0.75 & 2.5 & 0.1 & 0.2 & 52,53 \\
\hline & spha $^{e}$ & $-200^{a}$ & & -125 & 0.75 & 2.5 & & 0.1 & 0.08 \\
\hline L173 His $\rightarrow$ Leu & spha $^{e}$ & $-200^{a}$ & & -125 & 0.25 & 0.13 & 0.005 & 0.04 & 53 \\
\hline
\end{tabular}

${ }^{a}$ Initial values for calculation of other parameters. ${ }^{b}$ Calculated considering FC factor according to eq $14 .{ }^{c}$ References for the experimental oxidation potentials and the ET rates. ${ }^{d}$ Valid only if $\mathrm{B}_{\mathrm{A}}$ has not been affected by the mutation. ${ }^{e}$ Rb. sphaeroides. ${ }^{f}$ Rb. capsulatus. ${ }^{g}$ Rps. viridis.

TABLE 3: Experimental and Calculated Parameters for ET in the Inactive Branch

\begin{tabular}{|c|c|c|c|c|c|c|c|}
\hline mutation & species & $\begin{array}{c}\Delta \alpha \\
(\mathrm{meV})\end{array}$ & $\begin{array}{l}\Delta E_{(-)} \\
(\mathrm{meV})\end{array}$ & $C_{\mathrm{B}}^{2}$ & $\begin{array}{c}\left(V_{\mathrm{B}_{\mathrm{mut}}} / /\left(V_{\mathrm{AwTT}}\right)^{2}\right. \\
\text { calcd }^{a}\end{array}$ & $\begin{array}{c}k_{\mathrm{B}_{\mathrm{mul}}} / k_{\mathrm{AWT}} \\
\operatorname{expt}^{b}\end{array}$ & $\operatorname{ref}^{c}$ \\
\hline M210 Tyr $\rightarrow$ Phe & spha $^{d}$ & +65 & 110 & 0.4 & 0.02 & 0.004 & 65 \\
\hline M201 Gly $\rightarrow$ Asp & caps $^{e}$ & +100 & +100 & 0.36 & 0.04 & 0.04 & 49 \\
\hline
\end{tabular}

${ }^{a} V^{2}$ in the B branch of the mutant is divided by that in the A branch of the wild type. ${ }^{b}$ Calculated considering FC factor according to eq 14. ${ }^{c}$ References for the experimental oxidation potentials and the ET rates. ${ }^{d}$ Rb. sphaeroides. ${ }^{e}$ Rb. capsulatus.

TABLE 4: Matrix Element Calculations for the First Step of ET in the Wild Type RC

\begin{tabular}{|c|c|c|c|c|c|}
\hline authors, year, ref & method, basis & $\begin{array}{c}V_{1_{\mathrm{A}}} \\
(\mathrm{meV})\end{array}$ & $\begin{array}{c}V_{1_{\mathrm{B}}} \\
(\mathrm{meV})\end{array}$ & $V_{1_{\mathrm{A}}} / V_{1_{\mathrm{B}}}$ & species \\
\hline Warshel et al. $(1988)^{57}$ & semiempirical atomic coupl. & 0.83 & & & Rps. viridis \\
\hline Plato $(1991)^{13}$ & overlaps, SCF-AO & & & 2 & Rps. viridis \\
\hline Curry et al. $(1995)^{21}$ & parametrized pathway calc. & 5.3 & & & Rps. viridis \\
\hline Scherer et al. $(1995)^{8}$ & INDO/S, with extended tails & 7.5 & 2.5 & 3 & Rps. viridis \\
\hline Ivashin et al. $(1998)^{14}$ & ZINDO/S & $\begin{array}{l}2.4 \\
2.6\end{array}$ & 0.8 & 3 & $\begin{array}{l}\text { Rps. viridis } \\
\text { Rb. sphaeroides }\end{array}$ \\
\hline Zhang and Friesner $(1998)^{15}$ & ab initio $6-31 \mathrm{G}$ & 2.1 & 0.15 & 14 & Rps. viridis \\
\hline Kolbasov and Scherz (1999) & Kühn with modifications & $\begin{array}{l}7.6 \\
4.6\end{array}$ & $\begin{array}{l}2.9 \\
0.15\end{array}$ & $\begin{array}{l}2.6 \\
30\end{array}$ & $\begin{array}{l}\text { Rps. viridis } \\
\text { Rb. sphaeroides }\end{array}$ \\
\hline
\end{tabular}

$\mathrm{B}_{\mathrm{B}}$ system as a whole. This results in different distances between $\mathrm{P}$ and the accessory BChls in the two branches. The closest distance between the conjugated systems of these cofactors is about $0.7 \AA$ smaller in the A branch than in the B branch. In $R b$. sphaeroides there is no difference of the distances, but there is a pronounced difference between the relative positions of the acetyl groups of $\mathrm{P}_{\mathrm{B}}$ and $\mathrm{P}_{\mathrm{A}}$. (2) The asymmetrical distribution of electron density in $\mathrm{LUMO}(+)$. Apparently, the dependence of $V_{1}$ on this factor is similar in the two organisms (Figure 2) but shifted upward in Rps. viridis. This shift may reflect our simplified calculations of the orbitals of $\mathrm{P}$, in which the electron density on the vinyl group of $\mathrm{P}_{\mathrm{B}}$ may be underestimated.

Since the atomic structure of the bacterial RC had been published, several groups performed matrix element calculations. Table 4 compares the results of these calculations with ours. The nonunitary ratios between the $V$ values in the active and inactive branches of Rps. viridis were determined by all groups, although the absolute $V$ values were quite different. The larger $V_{\mathrm{A}}$ could be explained by the smaller distance between $\mathrm{P}$ and $\mathrm{B}_{\mathrm{A}}$, compared to that between $\mathrm{P}$ and $\mathrm{B}_{\mathrm{B}}$. However, the situation 
is not so simple in $R b$. sphaeroides where the distances are almost equal.

The calculated $V$ values and their decay rates with the donor/ acceptor distance strongly depend on the basis functions used in each calculation. Correctly chosen distance dependencies are of prime importance, since they determine the order of magnitude of the resulting coupling, whereas the exact treatment of the perturbation that mixes the functions is probably less important. The functions used by the different groups in calculating the overlap matrix elements are given in Table 4, and most of the functions decay fairly rapidly. Several reports have already discussed this issue. ${ }^{8,11,13,55-57}$ In the semiempirical calculations, the decay rate of the atomic wave function relies on the approximately exponential decay observed for long-range electron transfer. As depicted in Figure 3, the exponential decay does not hold for the distances that are relevant to the $\mathrm{P} / \mathrm{B}$ distances in bacterial RC. We have noted that the atomic matrix elements generally decay more slowly than the molecular ones. This discrepancy may also account for the relatively small $V$ values obtained, e.g., by Parson and Warshel. ${ }^{57}$ Ivashin et al., ${ }^{14}$ in their recent calculations, probably did not consider the tails of the wave functions and therefore could have underestimated the through-space couplings. This may be the reason their results depend, to a large extent, on the presence of the intermediate nonconjugated atomic groups that serve as bridges. In contrast, Scherer and Fischer used slowly decreasing expressions for the resonance integrals in their recent calculations ${ }^{8}$ and obtained large values for the matrix elements (Table 4). These values are very close to those obtained in the present study for Rps. viridis.

The calculated distance dependence of $V_{1}$ (Figure 3 ) deviates significantly from an exponential. It has a maximum near the native position of the molecules, and decays faster at long distances than at short ones. Interestingly, the experimental couplings in protein analyzed by Dutton et al. obey the same rule. ${ }^{58}$ These couplings could be better described by eq 1 with two different decay parameters, $\gamma$, for short and long distances, 0.9 and 2.05, respectively. The value of 1.4 for $\gamma$ published by Moser et al. ${ }^{20}$ is therefore the average value.

Another problem in calculating the values of $V$ concerns the identity of the primary donor; is it a supermolecule or two coupled monomers that interact separately with the accessory pigments? The small couplings obtained by our group and Zhang and Friesner ${ }^{15}$ for the inactive branch of $\mathrm{RC}$ were obtained only because $\mathrm{P}^{*}$ was treated as a single coherent state. The couplings of $\mathrm{B}_{\mathrm{B}}$ with the monomers of $\mathrm{P}$ have been added by considering their signs, according to eq 7. Apparently, the zero matrix element in the B branch is the result of canceling the couplings with the two monomers. If the monomers are independent, the squares of the couplings should be added into a positive, nonzero value. Furthermore, our calculations predict that for cancellation of $V_{\mathrm{B}}$, an equal participation of the monomers in the LUMO$(+)$ is required. If the absolute values of the coefficients $c$ in eq 7 were not equal, the summation of the individual coupling elements would be nonzero. Still, the relatively slow change of $V_{1_{\mathrm{B}}}$ with the value of $c_{\mathrm{B}}^{2}$, when $c_{\mathrm{B}}^{2}$ is around 0.5 , guarantees that $V_{1_{\mathrm{A}}} / V_{1_{\mathrm{B}}}$ remains very large. Interestingly, Zhang and Friesner ${ }^{15}$ also added equal contributions of $\mathrm{P}_{\mathrm{A}}$ and $\mathrm{P}_{\mathrm{B}}$ and obtained a relatively small value for $V_{1_{\mathrm{B}}}$.

The absolute values for the individual couplings of the two monomers of $\mathrm{P}$ with $\mathrm{B}_{\mathrm{A}}$ differ, and therefore their sum differs from zero. Here, the sum again depends on the relative contributions of $\mathrm{P}_{\mathrm{A}}$ and $\mathrm{P}_{\mathrm{B}}$ to the $\mathrm{LUMO}(+)$. The coupling of $B_{A}$ with $P_{B}$ is stronger than with $P_{A}$, so when the electron density shifts from $\mathrm{P}_{\mathrm{A}}$ to $\mathrm{P}_{\mathrm{B}}$, the matrix transfer element $V_{\mathrm{A}}$ increases. This makes $V_{\mathrm{A}}$ sensitive to changes of the polarization of the special pair and may account for the influence of the medium on the electronic coupling.

Experiments with mutated RCs support the dependence of $V_{\mathrm{A}}$ and $V_{\mathrm{B}}$ on the electronic distribution in the $\mathrm{LUMO}(+)$ of $\mathrm{P}$, in which the charge density has been shifted from one side to the other. Such mutations may introduce or remove hydrogen bonds and electric charges near $\mathrm{P}$ or $\mathrm{B}$. Mutations that change the number of hydrogen bonds with only one unit in $\mathrm{P}$ may show a clear and more distinct effect. The other mutations should alter the electrostatic field around the cofactors, and they are more complicated to consider. In this paper, we discuss two such mutations: M201/203 Gly $\rightarrow$ Asp $^{49,50}$ and M210 Tyr $\rightarrow$ Phe. ${ }^{59-61}$ Both mutations should influence the ET rate in the A and $\mathrm{B}$ branches (Tables 2 and 3).

When considering the M201 Gly $\rightarrow$ Asp mutation, we have followed the literature in assuming that an Asp residue under biological conditions is usually negatively charged. ${ }^{62}$ The negative charge should destabilize the electrons in P. However, according to the literature, the oxidation potential of $\mathrm{P}^{*}$ in the mutant is practically the same as in the wild type. Though there is some contradiction in the experimental data, we suppose that the Asp is ionized, as shown by Czarnecki et al. ${ }^{48} \mathrm{We}$ have assumed that because of the Asp interaction with $\mathrm{P}$, the electron density moved from $\mathrm{P}_{\mathrm{B}}$ to $\mathrm{P}_{\mathrm{A}}$. As mentioned above, we assume that the Asp substitution does not significantly affect the energy of $\mathrm{B}_{\mathrm{A}}{ }^{-}$, This assumption may be proven wrong in more accurate calculations. Yet, in the absence of accurate structural information we could not calculate the putative effect of this substitution and, therefore, did not try to estimate $\Delta G$ here. In calculating the electrostatic interaction of Asp M201 with P*, Asp M201 was positioned so that sterical hindrance of the Asp carboxylic residue was avoided. In our model, the distances between the Asp atoms and all the other atoms were all kept larger than 3 A. 63

Another mutation that might have an electrostatic effect on P involves substituting Phe for Tyr at M210 and thereby removing the dipole of the $\mathrm{OH}$ group. Having examined the $\mathrm{RC}$ atomic structure, neither hydrogen bond dissociation nor structural changes are expected to follow this mutation. Unfortunately, we cannot reliably predict the change of $\Delta G$ in the A branch using the redox potential of $\mathrm{P}$, because $\mathrm{B}^{-}$should be also strongly influenced. Alden et al. ${ }^{64}$ predicted an increase of $\Delta G$ in the mutant relative to the wild type because of destabilization of $\mathrm{B}_{\mathrm{A}}{ }^{-}$.

The satisfactory agreement between the calculated and experimental ET rates in the A branch for wild type and mutated RC (Table 2) supports the suggested significance of $V$ but does not rule out a significant contribution of $\Delta G$ to the observed changes of ET rates. It seems to us that in the B branch (Table 3 ) the changes of the ET rates depend more strongly on the matrix elements. Here, the coefficients $c$ in eq 7 change because of the changing electric field. Thus, the resultant electronic coupling becomes nonzero. As seen in Figure $1, V_{\mathrm{B}}$ in $R b$. sphaeroides increases to the first approximation linearly with polarization, and the rate constant increases as $V^{2}$. Consequently, at small polarizations, the rate constant in the $\mathrm{B}$ branch grows slowly.

Consider the energetics of the ET in the B branch in these mutants. ${ }^{49,65}$ Both mutation sites M201 and M210 are far from $\mathrm{B}_{\mathrm{B}}$ and probably do not influence its electronic energy levels. The oxidation potential in M201 Gly $\rightarrow$ Asp is the same as in the wild type, and in the other mutant it is $30 \mathrm{meV}$ more positive 
than in the wild type. Therefore, $\Delta G$ of the ET in the B branch of these mutants is probably the same or larger than in the wild type. Thus, the energetical changes cannot explain the increase of $k_{\mathrm{B}} / k_{\mathrm{A}}$ in the mutated $\mathrm{RC}$ unless one assumes that it slows down the ET from $\mathrm{P}$ to $\mathrm{B}_{\mathrm{A}}$.

\section{Appendix: Calculation of the Interatomic Resonance Integrals (after H. Kiihn ${ }^{26}$ )}

Each pairwise atom-atom matrix element is calculated as follows:

$$
S=\left\langle f_{\mathrm{D}}\left|H^{\prime}\right| f_{\mathrm{A}}\right\rangle
$$

where $f_{\mathrm{D}}$ and $f_{\mathrm{A}}$ are the one-electron wave functions of donor and acceptor atoms, respectively, $H^{\prime}$ is the perturbation Hamiltonian, equal to the approximate effective atomic potential in atomic units given by

$$
H^{\prime}(r)=-(1 / r)(Z-1 / r)
$$

where $r$ is the distance from the center of the nucleus and $Z$ is the effective charge of the atomic core. For carbon atoms, $Z$ equals 3.25 .

The wave function of the perturbing atom that is calculated only at small distances ( $r<R_{\pi}$, where $R_{\pi}$ is the van der Waals radius of a $\pi$-electron) from the atomic center can be represented by a Slater function (all equations are in atomic units)

$$
f_{\mathrm{A}}=N_{\text {slater }} r \exp (-0.5 \mathrm{Zr}) \cos \Theta
$$

where $\cos \Theta$ is the angular dependence of the atomic p-orbital;

$$
N_{\text {slater }}=\pi^{-0.5}(Z / 2)^{5 / 2}
$$

is a normalization constant.

The electron wave function at large distances $\left(r>R_{\pi}\right)$ from the atomic center is the solution of the Shrödinger equation for $U=-q^{2} /(D r)$, if the electron is in the field of the charge $q$. This is true for the following wave function (the parameters in eqs $\mathrm{A} 5-\mathrm{A} 8$ are in atomic units):

$$
f_{\mathrm{D}}=N r^{n-1} \exp (-a r) \cos \Theta
$$

$a=(2 I)^{1 / 2}=$ the distance decay factor, where $I$ is the ionization potential

$n=(a D)^{-1}=$ the effective quantum number for a neutral molecule

$$
N=N_{\text {slater }} R_{\pi}^{2-n} \exp \left\{(a-Z / 2) R_{\pi}\right\}
$$

The exponential dependence on $r, \exp (-a r)$, in the vicinity of the acceptor's atoms can be approximated as a linear dependence on $x_{j}, \exp \left(-a d_{i j}\right)\left(1-a x_{j}\right)$, where $d_{i j}$ is the distance between the donor's and acceptor's atoms and $x_{j}$ is the coordinate with zero on the acceptor's atom and its axis directed from the donor to the acceptor's atom. Substituting (A2-A8) in (A1) and integrating numerically in the vicinity of the acceptor's atom within the sphere with a radius of $R_{\pi}$ gives the value for the interatomic resonance coupling element (in $\mathrm{eV}$ ) as a function of the interatomic distances (in $\AA$ ) and direction cosines of the atomic p-orbitals (axis $Z$ is directed from the $i$ th atom toward the $j$ th atom),

$$
\begin{array}{r}
S_{i j}=13.6 a 3.04^{2-n} \exp \left(-a d_{i j}\right)\left(1.89 d_{i j}\right)^{n-1}\left\{-\cos Z_{i} \cos Z_{j}+\right. \\
\left.\left(a d_{i j}\right)^{-1}\left(\cos X_{i} \cos X_{j}+\cos Y_{i} \cos Y_{j}\right)\right\} \text { (A9) }
\end{array}
$$

Acknowledgment. This study was supported by the AvronMinevra center for photosynthesis and by the Deutsche Forschungsgemeinschaft, DFG-140, grant. We greatly appreciate helpful discussions with Prof. J. R. Norris from the Department of Chemistry, University of Chicago, and Prof. W. W. Parson from the Department of Biochemistry, University of Washington.

\section{References and Notes}

(1) Kirmaier, C.; Holten, D.; Parson, W. W. Biochim. Biophys. Acta 1985, 810, 49-61.

(2) Kellog, E. C.; Kolaczkowski, S.; Wasielewski, M. R.; Tiede, D. M. Photosynth. Res. 1989, 22, 47-59.

(3) Bylina, E. J.; Kirmaier, C.; McDowell, L.; Holten, D.; Youvan, D. C. Nature 1988, 336, 182-184.

(4) Ulstrup, J. Charge-Transfer Processes in Condensed Media; Springer-Verlag: Berlin, 1979; Vol. 10.

(5) Gunner, M. R.; Nichols, A.; Honig, B. J. Phys. Chem. 1996, 100, 4277-4291.

(6) Michel-Beyerle, M.-E.; Plato, M.; Deisenhofer, J.; Michel, H.; Bixon, M.; Jortner, J. Biochim. Biophys. Acta 1988, 932, 52-70.

(7) Sakuma, T.; Kashiwagi, H.; Takada, T.; Nakamura, H. Int. J. Quantum Chem. 1997, 61, 137-151.

(8) Scherer, P. O. J.; Scharnagl, C.; Fischer, S. F. Chem. Phys. 1995 197, 333-341.

(9) Parson, W. W.; Chu, Z. T.; Warshel, A. Biochim. Biophys. Acta 1990, 1017, 251-272.

(10) Scherer, P. O. J.; Fischer, S. F.; Lancaster, C. R. D.; Fritzsch, G.; Schmidt, S.; Arlt, T.; Dressler, K.; Zinth, W. Chem. Phys. Lett. 1994, 223 $110-115$.

(11) Plato, M.; Möbius, K.; Michel-Beyerle, M.-E.; Bixon, M.; Jortner, J. J. Am. Chem. Soc. 1988, 110, 7279-7285.

(12) Lendzian, F.; Huber, M.; Isaakson, R. A.; Endeward, B.; Plato, M.; Bönigk, B.; Möbius, K.; Lubitz, W.; Feher, G. Biochim. Biophys. Acta 1993, 1183, 139-160.

(13) Bixon, M.; Jortner, J.; Michel-Beyerle, M. E. Biochim. Biophys. Acta 1991, 1056, 301-315.

(14) Ivashin, N.; Källenbring, B.; Larsson, S.; Hansson, Ö. J. Phys. Chem. B 1998, 102, 5017-5022.

(15) Zhang, L. Y.; Friesner, R. A. Proc. Natl. Acad. Sci. U.S.A. 1998 95, 13603-13605.

(16) Stowell, M. H.; McPhillips, T. M.; Soltis, S. M.; Rees, D. C.; Abresh, E.; Feher, G. Science 1997, 276, 812-816.

(17) Deisenhofer, J.; Epp, O.; Sinning, I.; Michel, H. J. Mol. Biol. 1995, 246, 429-457.

(18) Bernstein, F. C.; Koetzle, T. F.; Williams, G. J. B.; Meyer, E. F.; Rodgers, J. R.; Kennand, O.; Simanouchi, T.; Tasumi, M. J. Mol. Biol. 1977, 112, 535-542.

(19) Hoffmann, R. Acc. Chem. Res. 1971, 4, 1-9.

(20) Moser, C. C.; Warnche, K.; Farid, R. S.; Dutton, P. L. Nature 1992, $355,796-802$.

(21) Curry, W. B.; Grabbe, M. D.; Kurnikov, I. V.; Skourtis, S. S.; Beratan, D. N.; Regan, J. J.; Aquino, A. J. A.; Beroza, P.; Onuchic, J. N. J. Bioenerg. Biomembr. 1995, 27, 285-293.

(22) Warshel, A.; Parson, W. J. Am. Chem. Soc. 1987, 109, 61436152.

(23) Alden, R. G.; Johnson, E.; Nagarajan, V.; Parson, W. W. J. Phys Chem. B 1997, 101, 4667-4680.

(24) Siders, P.; Cave, R. J.; Marcus, R. A. J. Chem, Phys. 1984, 81, 5613-5624

(25) Jaquet, R.; Schnupf, U. Chem. Phys. 1992, 165, 287-295.

(26) Kühn, H. Phys. Rev. A 1986, 34, 3409-3425.

(27) Libit, L.; Hoffmann, R. J. Am. Chem. Soc. 1974, 96, 1370-1383.

(28) Salem, L. The Molecular Orbital Theory of Conjugated Systems; Benjamin: New York, 1966.

(29) Petke, J. D.; Maggiora, G. M.; Shipman, L. L.; Christofersen, R. E. Photochem. Photobiol. 1980, 32, 399-414.

(30) Parson, W. W. Personal communication.

(31) Lin, X.; Murchison, H. A.; Nagarajan, V.; Parson, W. W.; Allen, J. P.; Williams, J. C. Proc. Natl. Acad. Sci. U.S.A. 1994, 91, 10265-10269. (32) Moss, D. A.; Leonhard, M.; Bauscher, M.; Mäntele, W. FEBS Lett 1991, 283, 33-36. 
(33) Gao, J.-L.; Shopes, R. J.; Wraight, C. A. Biochim. Biophys. Acta 1990, 1015, 96-108.

(34) Watanabe, T.; Kobayashi, M. Electrochemistry of chlorophylls. In Chlorophylls; Scheer, H., Ed.; CRC Press: Boca Raton, 1991; pp 287316.

(35) Trasatti, S. Pure Appl. Chem. 1986, 58, 955-966.

(36) Warshel, A.; Chu, Z. T.; Parson, W. W. J. Photochem. Photobiol. A: Chem. 1994, 82, 123-128.

(37) Warshel, A.; Chu, Z. T.; Parson, W. W. Chem. Phys. Lett. 1997, $265,293-296$.

(38) Thompson, M. A.; Zerner, M. C.; Fajer, J. J. Phys. Chem. 1991 $95,5693-5700$

(39) Scherer, P. O. J.; Fischer, S. F. Quantum Calculations for Special Pair Dimer and for Heterodimers: Analysis of the Internal Charge Transfer States. In The reaction center of photosynthetic bacteria. Structure and Dynamics; Michel-Beyerle, M.-E., Ed.; Springer-Verlag: Berlin, 1995; pp $89-104$

(40) Artz, K.; Williams, J. C.; Allen, J. P.; Lendzian, F.; Rautter, J.; Lubitz, W. Proc. Natl. Acad. Sci. U.S.A. 1997, 94, 13582-13587.

(41) Scherer, P. O. J.; Fischer, S. F. Chem. Phys. 1989, 131, 115-127.

(42) Huber, M.; Doubinskii, A. A.; Kay, C. W. M.; Möbius, K. Appl. Magn. Reson. 1997, 13, 473-485.

(43) Huber, M. Photosynth. Res. 1997, 52, 1-26.

(44) Marcus, R. A. Pure Appl. Chem. 1997, 69, 13-29.

(45) Marcus, R. A.; Sutin, N. Biochim. Biophys. Acta 1985, 811, 265322.

(46) Volk, M.; Aumeier, G.; Langenbacher, T.; Feick, R.; Ogrodnik, A.; Michel-Beyerle, M. E. J. Phys. Chem. B 1998, 102, 735-751.

(47) Sim, E.; Makri, N. J. Phys. Chem. B 1997, 101, 5446-5458.

(48) Czarnecki, K.; Kirmaier, C.; Holten, D.; Bocian, D. F. J. Phys. Chem. A 1999, 103, 2235-2246.

(49) Heller, B. A.; Holten, D.; Kirmaier, C. Science 1995, 269, 940945.
(50) Williams, J. C.; Alden, R. G.; Murchison, H. A.; Peloquin, J. M.; Woodbury, N. W.; Allen, J. P. Biochem. 1992, 31, 11029-11037.

(51) Steffen, M. A.; Lao, K.; Boxer, S. G. Science 1994, 264, 810816.

(52) McDowell, L. M.; Kirmaier, C.; Holten, D. Biochim. Biophys. Acta 1990, 1020, 239-246.

(53) McDowell, L. M.; Gaul, D.; Kirmaier, C.; Holten, H.; Schenck, C. C. Biochem. 1991, 30, 8315-8322.

(54) Arlt, T.; Bibikova, M.; Penzkofer, H.; Oesterhelt, D.; Zinth, W. J. Phys. Chem. 1996, 100, 12060-12065.

(55) Fischer, S. F.; Scherer, P. O. J. Chem. Phys. 1987, 115, 151-158.

(56) Parson, W. W.; Warshel, A. J. Am. Chem. Soc. 1987, 109, 61526163.

(57) Warshel, A.; Creighton, S.; Parson, W. W. J. Phys. Chem. 1988 92, 2696-2701

(58) Dutton, P. L. Personal communication.

(59) Nagarajan, V.; Parson, W. W.; Gaul, D.; Schenck, C. Proc. Natl. Acad. Sci. U.S.A. 1990, 87, 7888-7892.

(60) Nagarajan, V.; Parson, W. W.; Schenck, C. C. Biochem. 1993, 32, $12324-12336$.

(61) Finkele, U.; Lauterwasser, C.; Zinth, W.; Gray, K. A.; Oesterhelt, D. Biochem. 1990, 29, 8517-8521.

(62) Branden, C.; Tooze, J. Introduction to Protein Structure.; Garland: New York, 1991.

(63) Flory, P. Statistical Mechanics of Chain Molecules.; Wiley: New York, 1969

(64) Alden, R. G.; Parson, W. W.; Chu, Z. T.; Warshel, A. J. Phys. Chem. 1996, 100, 16761-16770.

(65) Gray, K. A.; Wachtveitl, J.; Oesterhelt, D. Eur. J. Biochem. 1992, 207, 723-731. 